\title{
Experimental study of ultrasound excited lock-in thermography
}

Wang Yang, Liu Hui and Liu Junyan School of Mechatronics Engineering, Harbin Institute of Technology, Harbin, 15001, P.R.China

Corresponding Author: Liu Hui Ph.D, School of Mechatronics Engineering, Harbin Institute of Technology, 422 Postbox, Harbin, Post Zip: 150001 , P.R.China, Tel: $86+451+86414557$ or $86+451+86413257$ Email: liuhuiamuer@sina.com or huiliuamuer@yahoo.cn

\section{Abstract}

Ultrasonic excited lock-in thermography (ULT) is a novel efficient nondestructive testing (NDT) technique. When amplitude modulated ultrasound waves are launched into sample, defects are heated up selectively. Effects of inhomogeneous heating can be suppressed compared to optical excited lock-in thermography (OLT). Signal to noise ratio is improved obviously. Thus sensitivity and detectability of defect are enhanced.

In this paper Experiments are carried out to inspect samples with fatigue cracks and effects of modulated frequency are investigated by ULT. The mechanism of heat generation is demonstrated. Specific optimized frequency can be acquired in different depth of defects with the same size. The relationship between modulated frequency and depth is analyzed. This experimental research is helpful for quantitative approach in NDT.

Keywords Nondestructive testing lock-in thermography ultrasonic excited, modulated frequency

\section{Appends}

The Fig. 1 shows the structure of artificial defects specimen

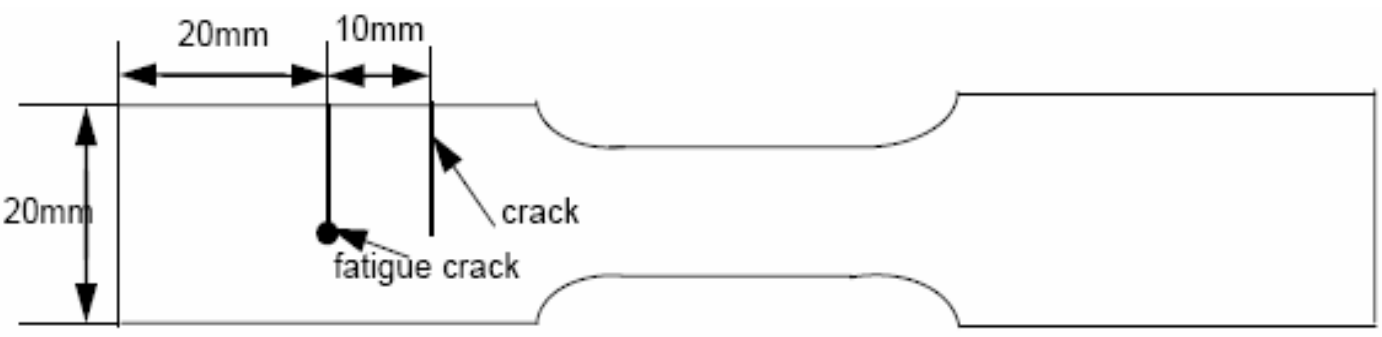

(a)

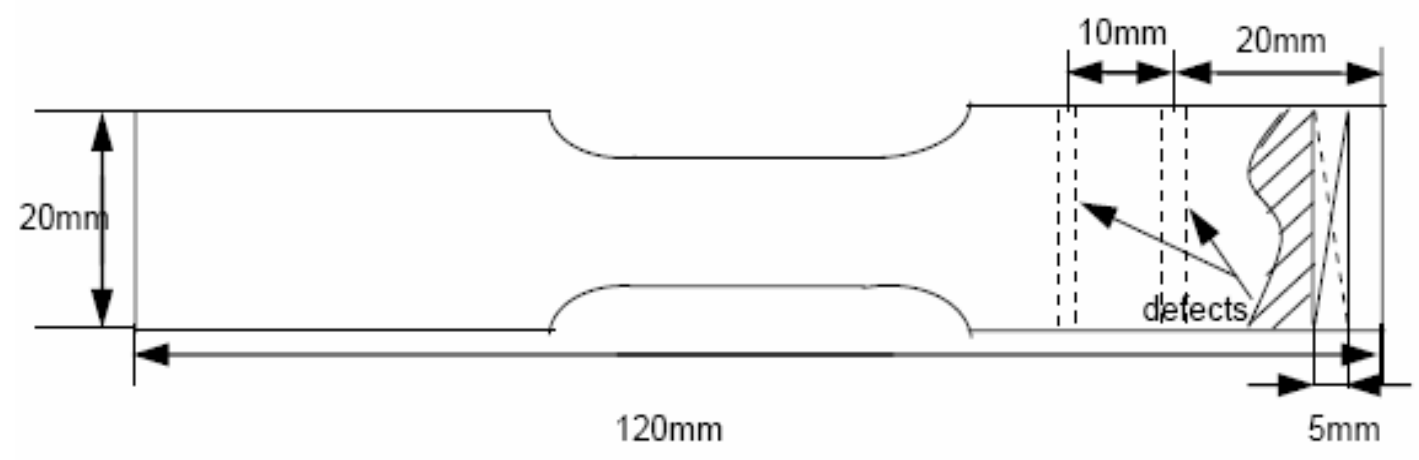

(b)

Fig.1 The structure of specimen

The Fig.2 shows the Cedip Lock-in thermography system and excited source of modulated ultrasound. 


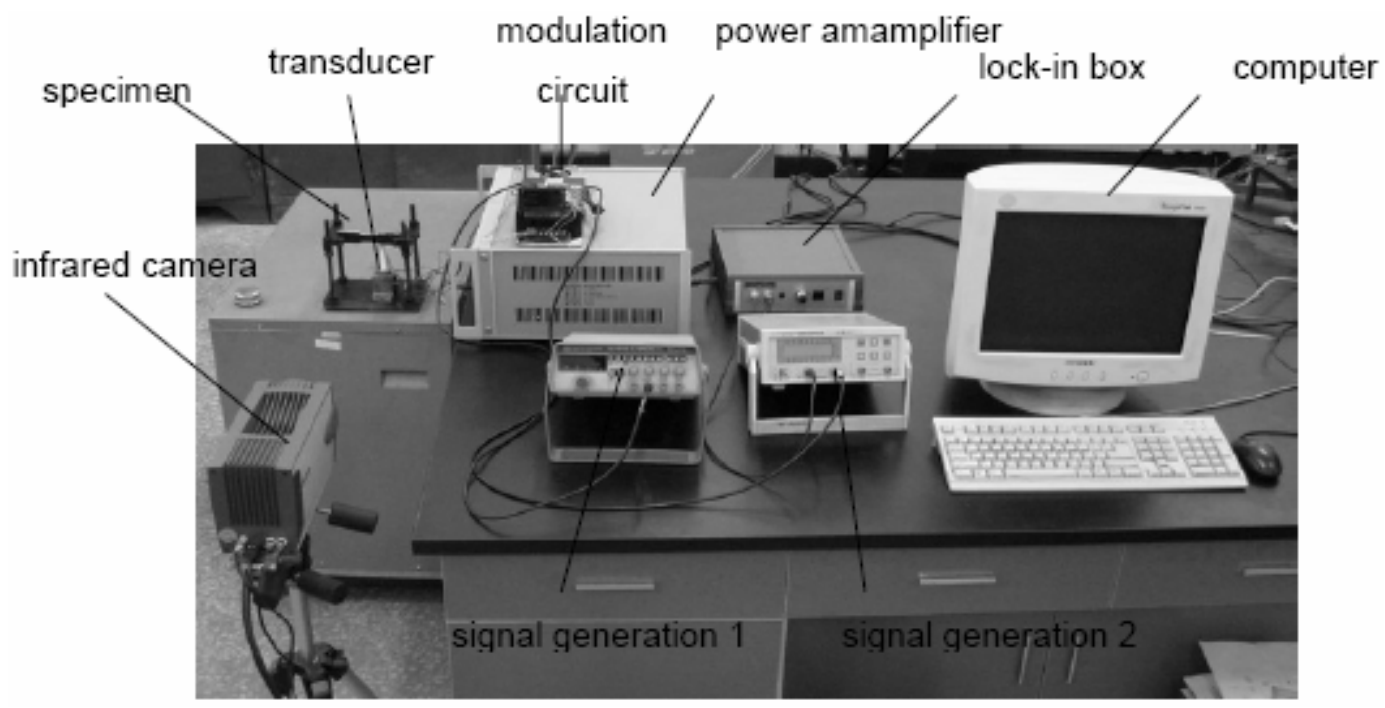

Fig 2 experimental setup

The Fig. 3 shows amplitude modulated ultrasonic excitation method and ultrasound excitation generates thermal waves in the defect.
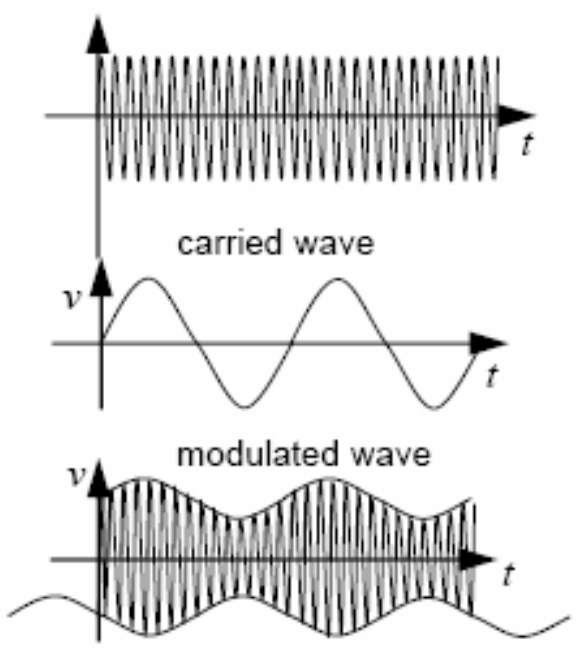

Amplitude modulated
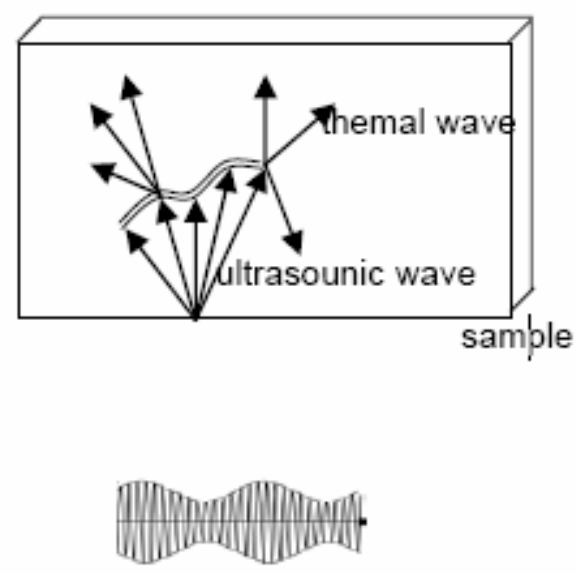

Modulated excited source

Fig 3 principle of modulated ultrasound excited

The experiment was performed in a large room and the temperature was about 20. Infrared camera of CEDIP Infrared Systems was fixed with a distant of $0.5 \mathrm{~m}$, facing the specimen perpendicularly

\section{Results}

Fatigue cracks are detected using modulated ultrasound excited lock-in thermography. Crack tips appear clearer in the image. It is analyzed that the absorption of modulated ultrasonic and the generation of periodical thermal wave are resulted from friction effects and stress concentration of defects.

Through changing modulated frequency, phase angle images are obtained from phase image sequences when defect is in certain depth. The relation between phase angle differences and modulated frequency is obtained for specific type and depth of defect by ULT.

Experimental results show that there exists an optimized modulated frequency for maximum phase differences at a certain depth of the defect. However, when the depth of defect is increased up to certain value, the maximum phase angle differences of defect and non-defect areas are too small to being detected. 